\title{
ESTRÉS LABORAL Y SUS EFECTOS PSICOSOMÁTICOS EN EL PERSONAL OBRERO DEL MINISTERIO DE TRANSPORTE Y OBRAS PÚBLICAS "MTOP" ZONAL 4
}

\author{
Jonathan Efren Pico Arreaga ${ }^{1 *}$, Guadalupe Del Rosario Bravo Cedeño ${ }^{1}$ \\ ${ }^{1}$ Facultad de Ciencias Humanísticas y Sociales, Carrera de Psicología Clínica, Universidad Técnica de Manabí, Portoviejo-Ecuador \\ * Autor para correspondencia: jpic04352@utm.edu.ec \\ Recibido: 2020/07/16 \\ Aprobado: 2020/11/20 \\ DOI: https://doi.org/10.26621/XVI23.2020.12.A07.PUCESI.2550.6684
}

\section{RESUMEN}

El estrés laboral ha constituido uno de los temas más importantes a tratar dentro del ambiente laboral latinoamericano, debido a que es un factor que tiende a influir en el desempeño de los trabajadores; y, por consiguiente, en el producto 0 servicio que toda institución brinda al público. Por tal motivo, es necesario que las instituciones mantengan un control de la salud mental de los empleados mediante la búsqueda de factores que puedan favorecer la aparición de estrés. Específicamente, en el Ministerio de Transporte y Obras Públicas (MTOP), siendo la principal institución encargada de la elaboración de la obra pública ecuatoriana, es crucial mantener un correcto ambiente laboral, para que sus trabajadores gocen de la misma manera de una óptima salud mental. Por ello, la investigación buscó evaluar los niveles de estrés laboral presentes en los trabajadores obreros de dicha institución, además de esclarecer aquellas variables que favorecen la aparición de estrés; para ello, se utilizó el cuestionario de problemas psicosomáticos de Hock, el cual permite establecer de manera rápida y eficaz los niveles de estrés presentes en la población, la cual fue constituida por 63 obreros; mediante el análisis descriptivo correlacional se pudo evidenciar que el $68 \%$ de la muestra presentó niveles de estrés, entre los cuales solo un 6\% mantenía un estrés de nivel medio. Por otra, parte se pudo evidenciar que factores como el estar casado, el tener hijos y el mantener sintomatología relacionada con ansiedad o depresión son variables predisponentes o predictores de la presencia de estrés laboral y este a su vez de ansiedad y depresión.

Palabras clave: estrés laboral, obreros, síntomas psicosomáticos, ansiedad, depresión

\section{ABSTRACT}

Job stress has been one of the most important issues to deal with in Latin American work environment, since it is a factor that affects an influence on the performance of workers, and, as regards the product or service that every institution provides the public. For this reason, institutions must maintain an optimal work environment for their proper functioning. Specifically, in the MTOP, being the main institution in charge of preparing the Ecuadorian public works, it is crucial to maintain a correct work environment, so that its workers enjoy optimal mental health in the same way. For them, the research sought to evaluate the levels of occupational stress present in the workers of the institution, for this, the Hock Psychosomatic Problems Questionnaire can be analyzed, which allows to establish quickly and effectively the levels of stress present in the population, which was made up of 63 workers, of whom $68 \%$ of the sample presented stress levels, among which only $6 \%$ maintained medium-level stress. On the other hand, it was possible to show that factors such as being married, having children and maintaining symptoms related to anxiety or depression they are predisposing variables or predictors of the presence of work stress.

Keywords: work stress, workers, psychosomatic symptoms, anxiety, depression 


\section{INTRODUCCIÓN}

En el mundo actual, los obreros son parte fundamental del ambiente productivo industrial de todo país; así, gracias a la revolución industrial, los obreros (también denominados "proletarios") han ido tomando el ambiente laboral de los países debido a su enorme aporte laboral, económico y productivo. Es por ello que, en los países industrializados y en vía de desarrollo, es fundamental contar con una capacidad obrera fuerte y óptima.

Dentro de Ecuador, los obreros han sido establecidos como trabajadores operativos o manuales, encargados de tareas centrales relacionadas con operación de maquinaria, tareas manuales de fuerza o elaboración de productos de manera manual. Han sido en gran parte los obreros quienes han llevado al desarrollo productivo industrial y nacional, llegando a ser parte esencial del crecimiento económico del país en al menos las últimas dos décadas.

A pesar de que los obreros actualmente sean parte fundamental del mecanismo productivo nacional, dicho sector tuvo un arduo camino hasta ser reconocidos como un sector indispensable del desarrollo productivo y económico del Ecuador. No fue sino hasta 1938 cuando fueron tomados realmente en cuenta en el panorama social y político, gracias a la conformación de la Confederación Ecuatoriana de Obreros Católicos y posteriormente en 1944 con la formación de la Confederación de Trabajadores Ecuatorianos, la cual impulsó la aprobación del Código de Trabajo. (Milk, 1997)

Sin embargo, a pesar de ser un sector productivo ya establecido en la sociedad, los obreros no dejan de ser un sector caracterizado por escasas garantías laborales, poco apoyo gubernamental, baja remuneración y escasa influencia en el ambiente político nacional. De este modo, no obstante se ha impuesto nuevas normativas que benefician al trabajador obrero, como menciona Paz y Miño (2016) estas no han sido en las dimensiones correctas que impongan en cierta medida la primacía del trabajo por encima del capital.

El pertenecer a un sector productivo tan infravalorado, sumado a las condiciones laborales a las que suelen estar sujetos, conforma una carga extra en los obreros además de la carga laboral a la están expuestos a diario. Todo ello, de acuerdo a los estudios de Oscco y colaboradores (2019), puede predisponer a los individuos a generar más estrés de lo habitual.

Es evidente que un ambiente laboral inadecuado es clave para el padecimiento de estrés laboral. Según datos de la Organización Internacional del Trabajo (OIT, 2016) en el continente, cerca del 30\% de la población laboral puede tener sintomatología relacionada con el estrés laboral. Estos datos nos permiten identificar la gravedad de esta problemática, que pocas veces es atendida por las autoridades.

Lo anteriormente mencionado demuestra que, dadas las condiciones laborales a la que están expuestos los trabajadores ecuatorianos, y hablando específicamente de los obreros, podemos establecer que el sector obrero nacional es propenso a sufrir de estrés laboral, lo que puede además (según datos de Roos y Sluiter) desembocar en otros trastornos como la ansiedad y depresión. Esto nos lleva a pensar, que la intervención en el ambiente laboral en materia de salud mental es necesaria, no solo por el correcto desempeño de las entidades, sino también por precautelar la salud de los trabajadores. (2004)
Dado que el estrés laboral es actualmente uno de los riesgos laborales más comunes en los trabajadores de la construcción (Carcaño, 2017), es importante la detección de casos de estrés laboral en las instituciones tanto públicas como privadas donde se encuentre laborando personal obrero, con la intención de realizar una detección temprana de dichos casos y que a su vez estos sean atendidos, de ser necesario, de la forma más inmediata posible para prevenir la transformación del estrés en otros trastornos de mayor complejidad.

Hablando específicamente de Ecuador, la población obrera de la construcción es una población que comúnmente se considera como no regulada, ya que, si estos están sujetos a construcciones a gran escala y por medio de empresas de construcción, los obreros suelen trabajar de forma irregular o precaria y bajo sus propias condiciones económicas y de seguridad. Sin embargo en instituciones como el Ministerio de Transporte y Obras Públicas (MTOP), al ser una institución del estado, esta debe llevar a cabo todos los requerimientos de ley para con sus trabajadores. El MTOP es una de las instituciones públicas que cuenta con personal obrero encargado de las tareas de operatividad y construcción. (Ministerio de Transporte y Obras Públicas, 2008). El MTOP es una institución pública que cuenta varios distritos ubicados en urbes centrales de cada provincia. Específicamente, en la ciudad de Portoviejo podemos encontrar al distrito zonal 4 del MTOP, que cuenta con un total de 63 obreros, donde se puede encontrar a personal encargado de labores de construcción, manejo de maquinaria, manejo de vehículos de transporte de personal y personal encargado del estudio de suelo.

Al ser una población que, como se ha mencionado, está expuesta a factores psicosociales que puedan generar un ambiente perjudicial para la salud mental, es de suma importancia hallar datos que puedan permitir establecer el panorama real que mantienen los obreros del MTOP en la ciudad de Portoviejo; estos a su vez permitirán establecer la población que mantiene sintomatología relacionada con el estrés, y al mismo tiempo, el presente estudio podría servir a las entidades pertinentes para tomar medidas que permitan mejorar el ambiente laboral de los trabajadores de la construcción.

Por lo mencionado con anterioridad, la presente investigación va destinada a la detección de sintomatología relacionada con el estrés laboral en el personal obrero del Ministerio de Transporte y Obras Públicas, distrito Dirección Zonal 4, distrito Portoviejo.

\section{MATERIALES Y MÉTODOS}

La presente investigación se basa (como no podría ser de otro modo) en la necesidad de obtener datos que muestren la veracidad de la situación real que se evalúa. En este caso, el estudio se lleva al cabo con un carácter cuantitativo que permita visualizar la presencia de estrés laboral, además de ser de carácter descriptivo ya que evalúa los niveles de estrés laboral en relación con otras variables como edad, sexo, estado civil y número de hijos. Para obtener los datos necesarios para la investigación se tomó en cuenta la aplicación de dos baterías de test.

Por un lado, se aplica el Cuestionario de Problemas Psicosomáticos de Hock, el cual consiste en un total de 12 ítems en los que se describen los síntomas más habituales asociados al estrés y en los que el trabajador debe señalar la frecuencia referente a los últimos 3 meses; dicho cuestionario permitió identificar el estrés laboral en diferentes niveles de intensidad a través de la frecuencia de síntomas psicosomáticos causados por el estrés laboral; la selección del mismo también permite establecer el nivel de estrés laboral sin ser intrusivo con el test paralelo que evalúa ansiedad y depresión. 
Por otra parte, y como medio para efectuar una adecuada correlación de variables de la investigación, se realizó la aplicación de la escala de Ansiedad y Depresión del Golberg, la cual nos permite determinar rasgos de ansiedad y depresión de una manera rápida, este cuestionario consiste en dos escalas, una de depresión y otra de ansiedad, cada uno con dos sub escalas de 4 y 5 preguntas.

Ambos test fueron adaptados a un cuestionario junto con las demás variables a evaluar, como edad, sexo, número de hijos, religión y estado civil. Cabe señalar que el cuestionario fue adaptado de forma que facilite la aplicación a la población descrita y que esta sea lo más sencilla y corta para la correcta recolección de datos. Asimismo, cabe destacar que ambos cuestionarios permiten su correlación.

En la presente investigación, se evaluó una población de 63 individuos, quienes comprenden la totalidad de obreros que laboran en el MTOP Distrito Zonal-4 en la ciudad de Portoviejo. Mediante la aplicación de las baterías de test ya descritas a la población censal establecida, se pudo establecer el vínculo entre los niveles de estrés y las variables planteadas como edad, sexo, estado civil, número de hijos, además de la correlación del estrés laboral con la presencia de rasgos de ansiedad y depresión.

\section{RESULTADOS Y DISCUSIÓN}

Como descripción de la población seleccionada, podemos visualizar en la Figura 1 y 2 la distribución de la misma por estado civil y número de hijos. Algo de importancia a destacar es que la presencia mujeres obreras es nula; esto se relaciona con el estudio realizado por Chávez (2014) donde se puede observar un porcentaje muy reducido de mujeres presentes en el sector. Ello nos lleva a interpretar los resultados del estudio de manera que no englobe a ambos sexos. Otro factor importante a recalcar es que la edad predominante en la población está comprendida entre los 30-39 años, tal y como se refleja en la Figura 3.

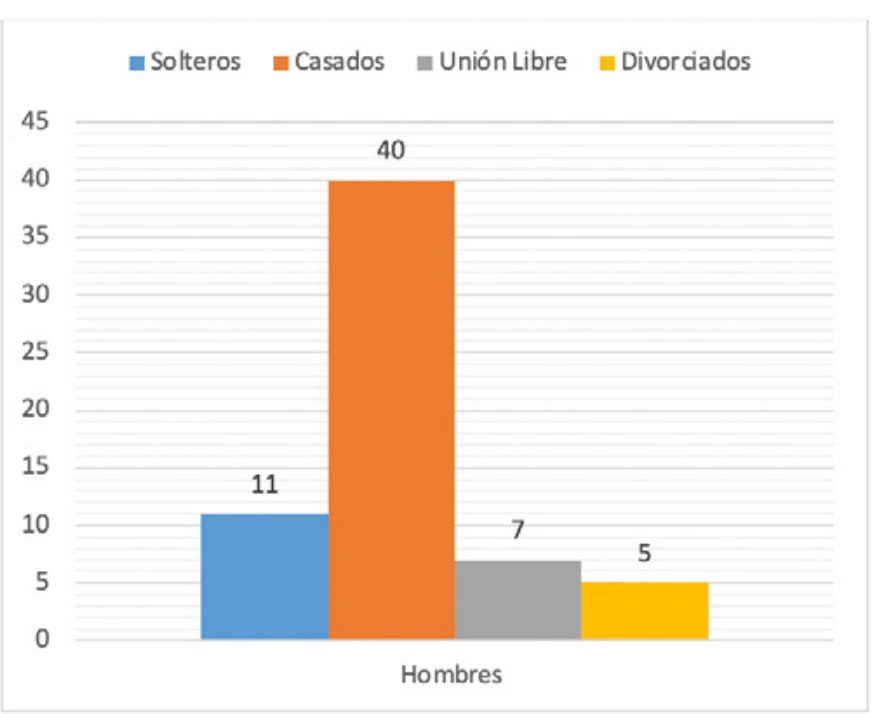

Figura 1. Distribución de la muestra en relación con su Estado Civil.

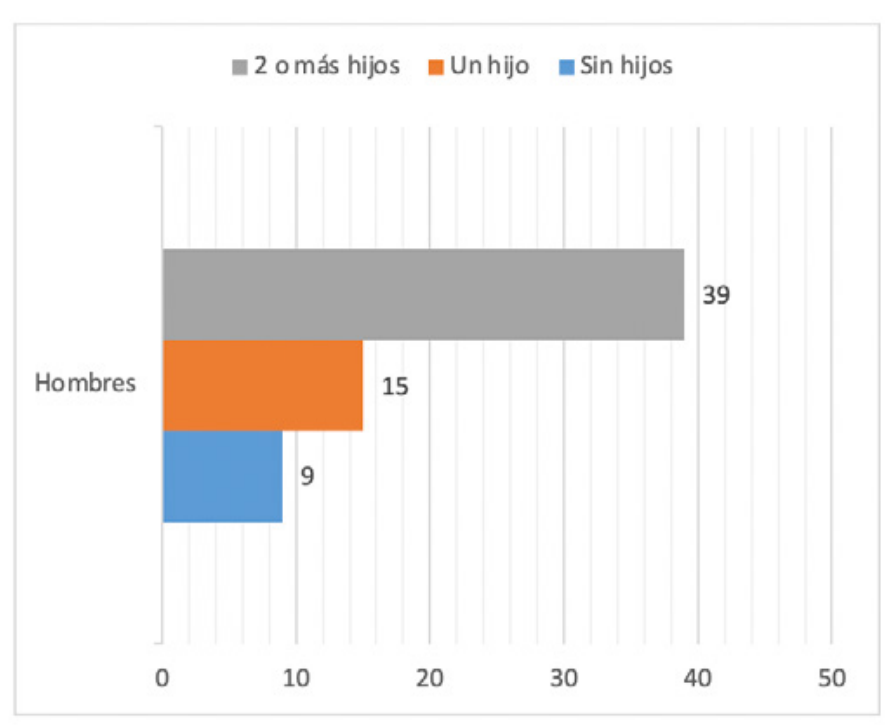

Figura 2. Distribución de la muestra en relación con número de hijos.

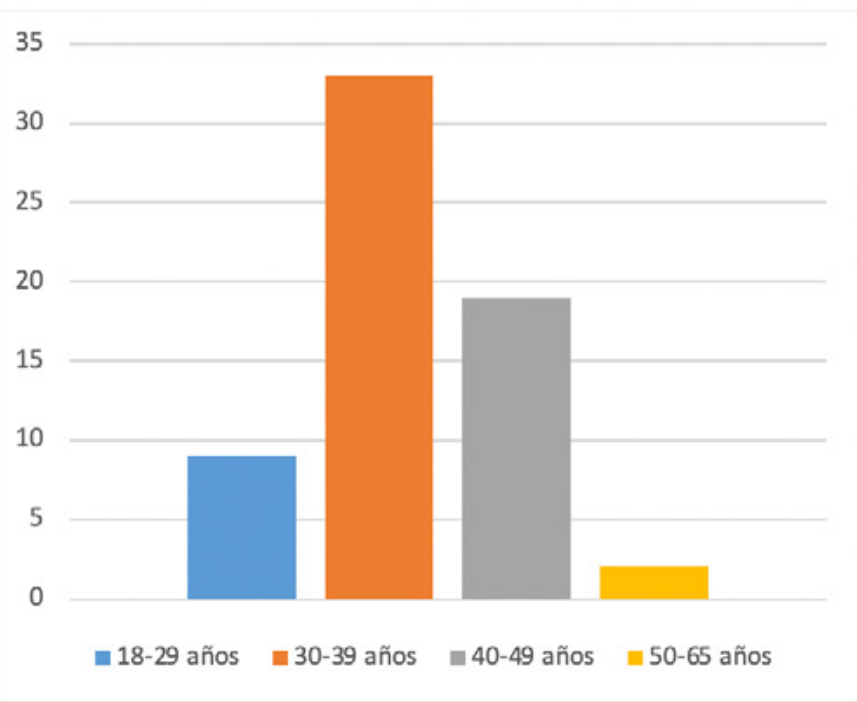

Figura 3. Distribución de la muestra en relación a la edad.

Como podemos observar en la Figura 4, los datos reflejan que la mayoría de la población cuenta con síntomas de estrés, sin embargo, el nivel de estrés que se evidencia es leve con un $62 \%$ de casos, el cual no significa sintomatología grave además de que no requiere un seguimiento 0 tratamiento inmediato, ya que el CPP recomienda simplemente en este nivel reconocer el entorno en el cual el individuo se encuentra y buscar modificarlo, debido a que prolongar este estado puede desequilibrar a la larga e influir en el ambiente de trabajo y social.

Por otro lado, un 32\% de la población no muestra sintomatología psicosomática, lo que, sumado a lo anteriormente mencionado, da evidencia que el estrés laboral no presenta un problema demasiado serio; sin embargo, la presencia de un $6 \%$ de estrés medio en la población es síntoma de que los casos con estrés leve pueden recaer en niveles de estrés con mayor intensidad. Además, la mera existencia de estrés de nivel medio requiere la asistencia profesional, dado que puede traducir un gran desgaste mental y físico que pueden convertirse en sintomatología más severa bajo ciertos parámetros. 


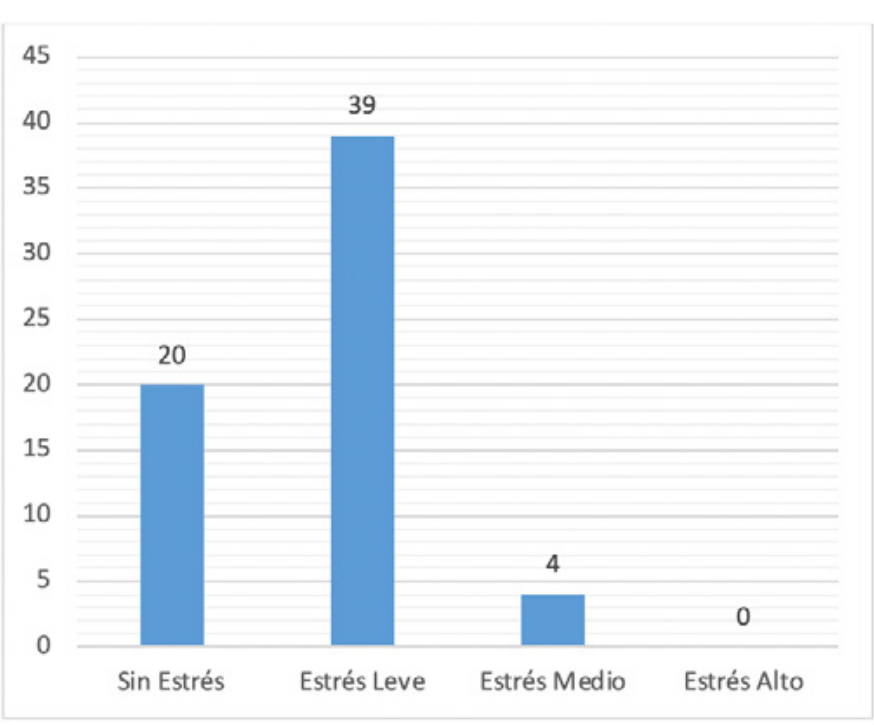

Figura 4. Distribución de la población por grados de estrés laboral.

En la relación entre estrés laboral de la población obrera y la edad, podemos observar en la Figura 4 que la edad predominante está entre los 30 y 39 años,g donde nuevamente la población con estrés laboral leve supera la mayoría del total comprendido. Otro dato a destacar es que los dos grupos de edad más jóvenes cuentan con niveles de estrés medio, lo que nos puede llevar a suponer que la población obrera menor a 39 años es más propensa a padecer estrés labor alto, dado que también ambos grupos de edad cuentan con pocos casos de personal sin estrés laboral. Sin embargo, dichos datos muestran disconformidad con los hallados por Chávez (2014), donde los obreros de mayor edad son más propensos a sufrir de estrés y enfermedades físicas.

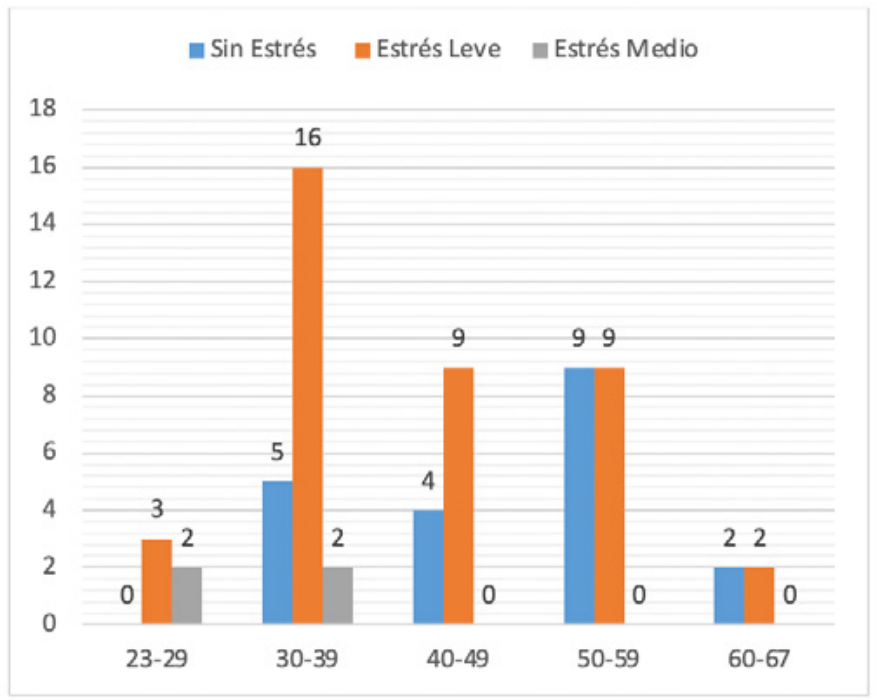

Figura 5. Distribución de la población obrera del MTOP por edades y niveles de estrés.

De acuerdo a los datos de la Figura 6, respecto al estrés laboral en relación con el estado civil, podemos destacar la presencia de 28 individuos que presentan estrés laboral, a lo que podríamos unir a los 7 obreros en situación de "unión libre", lo que nos lleva a suponer que el contar con un cónyuge o conviviente puede ser una variable a tomar en cuenta al evaluar estrés laboral. Lo anteriormente mencionado concuerda con los resultados de la población soltera y divorciada, lo a que su vez lleva a suponer la afirmación que el estado civil puede influir en la obtención de estrés laboral.

Respecto a los datos referentes al número de hijos en la Figura 7, po- demos destacar que el $87 \%$ de la población con presencia de estrés tiene al menos un hijo; esto, sumado a que los individuos sin hijos es la población predominante entre los que no muestran sintomatología psicosomática de estrés laboral, nos lleva a suponer que el número de hijos es una variable a tomar en cuenta y que posiblemente la carga familiar puede influir en la obtención de estrés laboral.

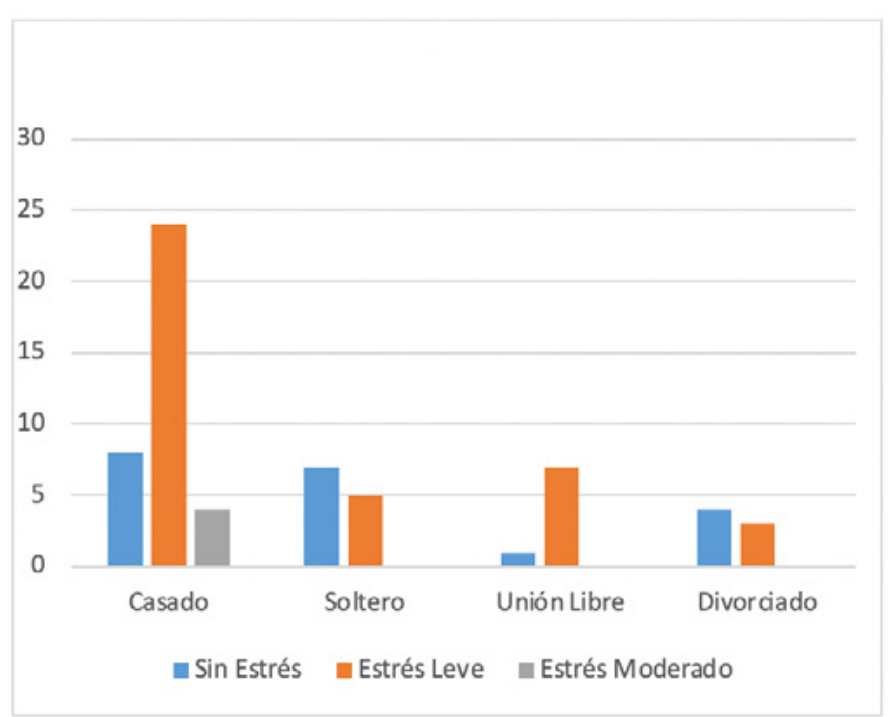

Figura 6. Distribución de la población obrera del MTOP por estado civil.

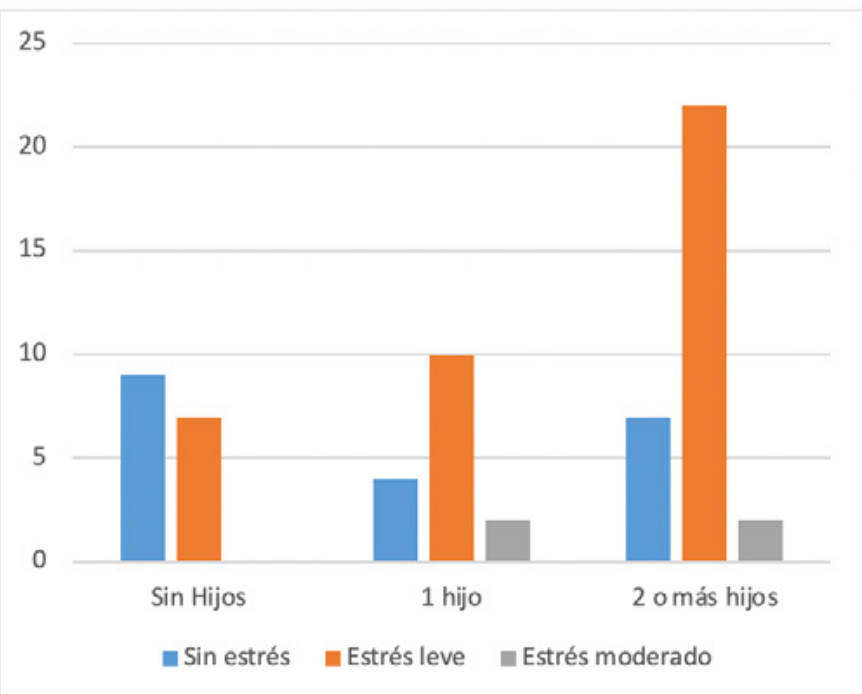

Figura 7. Distribución de la población obrera del MTOP por el número de hijos.

Mediante los datos obtenidos en la Figura 8, se puede observar cómo la diferencia cuantitativa entre la población sin rasgos de ansiedad frente a quienes no poseen dichos rasgos disminuye en proporción cuando se hace la diferenciación con individuos que registren sintomatología relacionada con el estrés laboral. Además de recalcar que la población con estrés supera cuantitativamente cuando se trata de niveles de estrés elevados, esto nos lleva a comprender que el nivel de estrés está ligado a la acumulación previa de ansiedad, que convierte al estrés "bueno" en estrés "malo" y lo cronifica.

Por otro lado, la Figura 9 nos muestra datos similares a lo anteriormente mencionado y nos demuestra que, cuando se busca rasgos de depresión en trabajadores con estrés laboral, estos suelen tener más presencia en los niveles elevados de estrés laboral, además de que la presencia de depresión alcanza su punto máximo en aquellos trabajadores con estrés 
medio, pues los 4 obreros que contaban con este nivel de estrés a su vez mantenían rasgos de depresión.

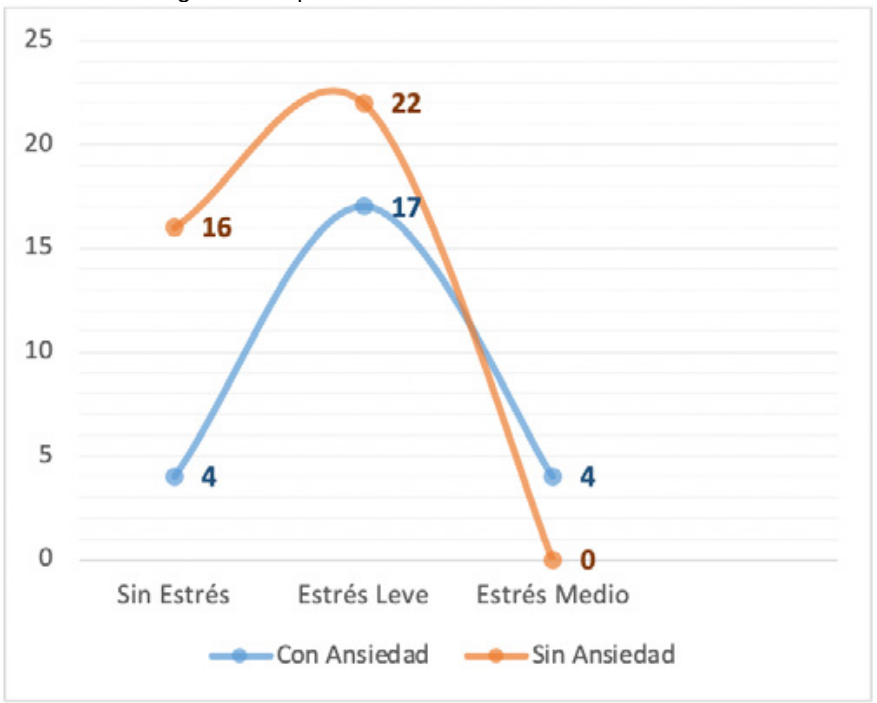

Figura 8. Distribución de la población evaluada con presencia de ansiedad.

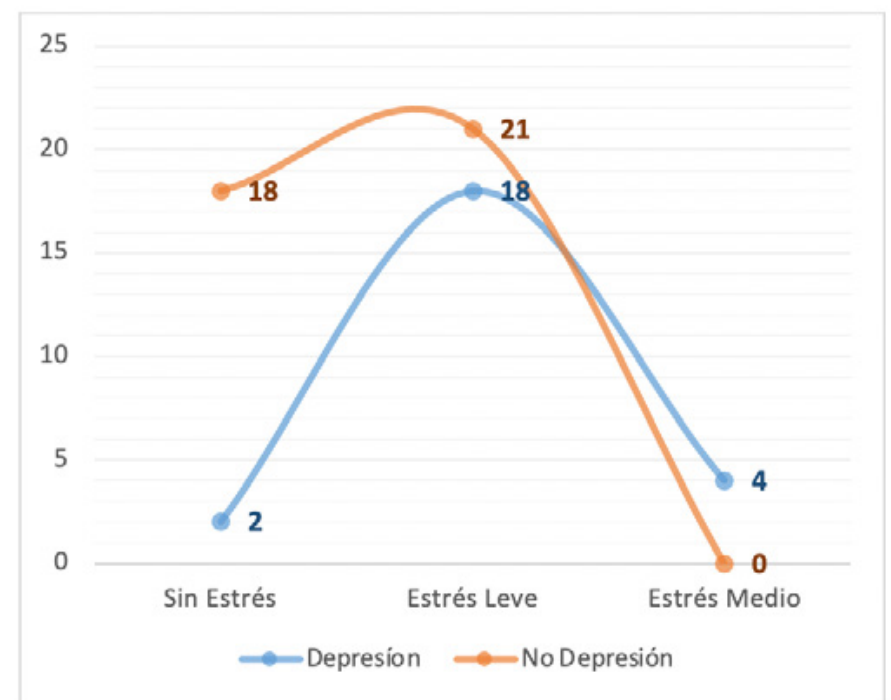

Figura 9. Distribución de la población evaluada con presencia de depresión.

Una vez concluido el análisis de los datos obtenidos en la investigación, podemos confirmar la presencia de sintomatología relacionada con el estrés laboral en los obreros del MTOP; así se pudo encontrar que el 62\% de la población padece estrés laboral en diferentes niveles, datos similares a los hallados por Estrada y Sánchez (2016), destacando el estrés leve como el predominante, sin embargo, se debe tomar en cuenta aquellos individuos que entraron en el nivel de estrés medio.

Estos datos muestran que el ambiente laboral de los obreros suele estar ligado a factores ambientales que pueden favorecer el desencadenamiento de estrés laboral. Tal y como lo menciona Holguín (2019) en sus conclusiones, los obreros se encuentran expuestos a diversos factores estresores los cuales les generan daños tanto físicos como mentales.

Otro de los datos a destacar es la reducida presencia de obreros con un nivel de estrés mayor al leve, ya que este no tiene una presencia significativa, lo que a su vez se ha relacionado con los datos mostrados por Estrada y Sánchez (2016). Todo ello lleva a suponer que los trabajadores de la construcción, a pesar de contar con lo requerido en su ambiente laboral para padecer estrés, no cuentan con condiciones suficientemente extenuantes como para que el estrés se muestre en niveles altos 0 que afecten de manera grave, lo que no necesariamente significa que el estrés laboral no sea un afección a tomar en cuenta en dichos ambientes laborales.

En cuanto a la correlación de la presencia de estrés laboral y la aparición de sintomatología relacionada con depresión, el estudio permitió identificar que más de la mitad de los evaluados con síntomas de estrés muestran presencia de depresión en diferentes niveles. Esto, junto a los resultados de Rodríguez, Tovalin, Gil, Salvador y Acle (2018), demuestran que la presencia de estrés laboral suele mostrar una coexistencia con síntomas depresivos.

Por otra parte, se establece un nexo con los estudios de Blanco y Thoen (2017), donde la presencia de ansiedad puede ser utilizada como un factor asociado al estrés laboral. Como se evidencia en los datos obtenidos, la presencia de ansiedad en los obreros con estrés laboral es de un $21 \%$, lo que no muestra tanta prevalencia como los datos de depresión. Esto demuestra que los trabajadores de la construcción pueden desencadenar ansiedad como resultado de la presencia de estrés en relación con el ambiente laboral.

\section{CONCLUSIONES}

Del estudio se puede concluir que el estrés laboral es una afección que puede presentarse en diferentes ámbitos laborales y ocupacionales, donde el área obrera de construcción no es la excepción; mediante los datos recabados podemos establecer que cerca del $68 \%$ de la población obrera del MTOP de la ciudad de Portoviejo presenta estrés laboral en diferentes niveles; estos a su vez pueden ser determinados o previstos por diferentes factores como la edad, estado civil, número de hijos, etc. Además de la presencia de sintomatología relacionada con la ansiedad 0 estrés.

Se ha podido establecer que la población femenina se mantiene reducida o inexistente tanto en este como en varios estudios dirigidos a la población obrera de la construcción, lo que no nos permite establecer una diferenciación entre los diferentes sexos y la presencia de estrés laboral; ello impide, por ende, establecer al sexo como un determinante al momento de evaluar estrés laboral.

Sin embargo, se puede establecer aquellas variables encontradas para determinar posibles casos de estrés laboral en la población obrera. Factores como el tener más de un hijo muestran una alta incidencia en la obtención de estrés laboral. Esto, sumado a que los individuos con cónyuges suelen tener más riesgo de adquirir estrés laboral, demuestra que los factores psicosociales y familiares son de gran importancia para el estudio de la adquisición del estrés laboral y sus factores desencadenantes, lo que deja la puerta abierta a futuros análisis donde se ponga en foco en aspectos de conciliación familiar.

Se pudo evidenciar que la presencia de rasgos de ansiedad, suelen estar ligados a una coexistencia de estrés laboral, tal y como se refleja en el estudio realizado por Buselli, Baldanzi y Cristaudo (2016) quienes mencionan que los síntomas de los trastornos de Ansiedad suelen coexistir con los niveles de estrés laboral en los trabajadores. De la misma manera, la depresión suele estar ligada a factores que predispongan a los trabajadores a obtener estrés laboral, tal y como lo describe Perales y colaboradores (2011) donde la depresión se muestra como la tercer patología con más presencia en la población laboral evaluada por los mismos. 


\section{REFERENCIAS BIBLIOGRÁFICAS}

Blanco, T., \& Thoen, M. (2017). Factores asociados al éstres laboral en policías penitenciarios costarricenses. Revista Costarricense de Psicología, 36(1), 45-59.

Buselli, R., Baldanzi, S., \& Cristaudo, A. (marzo de 2016). Trastornos de estrés laboral: variabilidad en la expresión clínica y dificultades en el diagnóstico psiquiátrico. La medicina del Lavoro, 2(107), 92-101. Obtenido de https://europepmc.org/article/ $\mathrm{med} / 27015025$

Cano, D. (2009). Regresión laboral. ¿Estado constitucional de derechos?, 291-314.

Carcaño, R. (Agosto de 2017). Riesgos psicosociales en trabajadores de la construcci\{on. Riesgos Laborales(2), 12-19.

Chávez, C. (2014). Estrés laboral en trabajadores de la construcción. Reviste de investigación cientifica UTE, 100-106.

de Roos, R., \& Sluiter, J. (2004). Depressie als beroepsziekte: Identificatie van werkgebonden psychosociale risicofactoren uit de landelijke registratie en een systematisch literatuuronderzoek. TBV-Tijdschrift voor Bedrijfs-en Verzekeringsgeneeskunde(12), 383-389.

Estrada, M., \& Sánchez, Y. (2016). Estrés Laboral en 15 Obreros de la construcción ARTYCO S.AS. de la ciudad de Medillín. Antioquia: Corporación Universitaria Minuto de Dios.

Holguín, M. (2019). Ingluencia del Éstres percibido sobre la satisfacción laboral en obreros de construcción civil en Lima metropolitana población. Lima: Universidad San Ignacio de Loyola.

Milk, R. (1997). Movimiento Obrero Ecuatoriano: El desafio de la integración. Quito: Digital Docutech.

Ministerio de Transporte y Obras Públicas. (2008). Ministerio de Transporte y Obras Públicas. Recuperado el 13 de diciembre de 2019, de Ministerio de Transporte y Obras Públicas Web Site: https://www.obraspublicas.gob.ec

OIT. (2016). Estrés en el Trabajo. Ginebra: Organización Internacional del Trabajo.

OMS. (1994). Global Strategy on Occupational Health for All. Ginebra: OMS.

Oscco, W., Sarcines, C., \& Torres, O. (2019). Condiciones laborales y nivel de estrés en enfermeros de un hospital público peruano. Revista Medica Panecea, 91-94.

Paz y Miño, J. (2016). Trabajadores y movimiento obrero. Elementos históricos para una economía politica sobre el trabajo en Ecuador. Historia y Economia, 1-24. Obtenido de http://the.pazymino. com/JPYMC-TRABAJADORES_Y_MOVIMIENTO_OBRERO.pdf

Perales, A., Chue, H., Padilla, A., \& Barahona, A. (2011). Estrés, ansiedad y depresión en magistrados de Lima, Peru. Revista Peruana de Medicina Experimental y Salud Publica, 581-588.

Rodríguez, M., Tovalin, J. H., Gil, P. R., Salvador, J., \& Acle, G. (2018). Trabajo emocional y estresores laborales como predictores de ansiedad y depresión en profesores universitarios mexicanos. Informació Psicológica. 115, 93-106. 